\title{
Influência da competição catiônica nos valores de fator de retardamento e coeficiente de dispersão-difusão de zinco e cobre no solo ${ }^{1}$
}

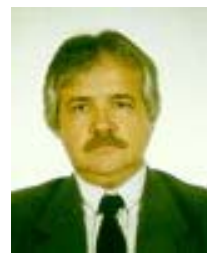

\author{
Mauro A. Martinez ${ }^{2}$, Victor B.N. Ramos ${ }^{3}$, Antônio T. de Matos ${ }^{4}$, Rubens A. de Oliveira ${ }^{5}$ \& Silvana N. da Costa \\ 1 Parte da dissertação de Mestrado do segundo autor, apresentada à UFV, parcialmente financiada pela FAPEMIG \\ 2 UFV. CEP 36571-000, Fone: (31) 3899-1910, Fax: (31) 3899-2735. E-mail: mmauro@mail.ufv.br (Foto) \\ ${ }^{3}$ DEA/UFV. Fone: (75) 252-1065 \\ 4 UFV. E-mail: atmatos@mail.ufv.br \\ 5 UFV. E-mail: ralves@mail.ufv.br \\ ${ }^{6}$ AMDE/PMCG. E-mail: silvanapa@uol.com.br
}

Protocolo $083-16 / 07 / 1999$

\begin{abstract}
Resumo: Objetivando-se avaliar os efeitos da competição catiônica nos valores do fator de retardamento $\left(f_{r}\right)$ e do coeficiente de dispersão-difusão (D) realizaram-se ensaios de mobilidade de metais em colunas de lixiviação submetidas a escoamento permanente e saturado. Foram aplicadas soluções de zinco (700 $\left.\mathrm{mg} \mathrm{L}^{-1}\right)$, cobre $\left(200 \mathrm{mg} \mathrm{L}^{-1}\right)$ e de zinco mais cobre, nas mesmas concentrações, para obtenção das respectivas curvas de eluição. Os materiais de solo utilizados foram retirados dos horizontes A, B e C de um Latossolo Vermelho-Amarelo, álico (LVa) e de um Podzólico Vermelho-Amarelo, câmbico fase terraço (PVc) coletados no município de Viçosa, MG. Nos solos estudados em todos os horizontes, o cobre apresentou maiores valores de fator de retardamento que o zinco, indicando apresentar maior interação com a fração sólida do solo. As baixas velocidades de avanço das soluções proporcionaram a obtenção de baixos valores de coeficiente de dispersão-difusão no solo e a competição catiônica diminuiu a capacidade de retenção dos cátions no solo.
\end{abstract}

Palavras-chave: metais pesados, deslocamento miscível, dispersão de poluentes

\section{Effect of the cationic competition on the retarding factor and dispersion-diffusion coefficient of zinc and copper in soil}

\begin{abstract}
The effects of the cationic competition on the retarding factor $\left(f_{r}\right)$ and the dispersion-diffusion coefficient (D) were evaluated with conducting metal mobility trials in leaching columns submitted to a saturated and steady flow. Solutions of zinc (700 $\left.\mathrm{mg} \mathrm{L}^{-1}\right)$, copper (200 $\mathrm{mg} \mathrm{L}^{-1}$ ) and zinc plus copper in the same concentrations, were used to obtain elution curves. Soil materials from the A, B and C horizons of Oxisol (LVa) and of Ultisol (PVC), collected in Viçosa, MG, were used. In all soil materials used, copper presented higher retarding factor values than zinc, which indicates a higher copper-soil interaction. The low velocities of the solutions resulted in low dispersion-diffusion coefficient values. Cationic competition decreased the soil retention capacity for the cations.
\end{abstract}

Key words: heavy metals, miscible displacement, pollutant dispersion

\section{INTRODUÇÃO}

A mobilidade de solutos no solo está inversamente relacionada à adsorção dos mesmos à fração sólida ou às condições do meio que favoreçam a precipitação dos íons (Matos et al., 1998). A adsorção iônica ao complexo de troca do solo faz com que os íons mantenham intercâmbio com a solução do solo, proporcionando ora sua retenção junto à fração sólida, ora sua disponibilização no meio aquoso.

A presença de cátions diversos na solução do solo proporciona a competição entre eles, pelos sítios de adsorção, favorecendo a lixiviação daqueles que não são retidos, fato que constitui um alerta quanto ao perigo de contaminação dos mananciais de água subterrânea, principalmente no que diz respeito aos metais pesados. Mitchell (1965) citado por Ferreira \& Cruz (1991) determinou a seguinte ordem de dificuldade de deslocamento de cátions adsorvidos aos minerais de argila: $\mathrm{Cu}>\mathrm{Pb}>\mathrm{Ni}>\mathrm{Co}>\mathrm{Zn}>\mathrm{Ba}>\mathrm{Rb}>\mathrm{Sr}>\mathrm{Ca}>\mathrm{Mg}>\mathrm{Na}>\mathrm{Li}$. Observa-se que, por esta seqüência, o cobre deve estar mais fortemente adsorvido ao solo que o zinco. Matos (1995) encontrou, para os solos do município de Viçosa, MG, a seguinte ordem de retenção de metais pesados: $\mathrm{Pb}>\mathrm{Cu}>\mathrm{Cd}>\mathrm{Zn}$. 
Campos \& Elbachá (1991) definem fator de retardamento como a capacidade de retenção ou efeito tampão do solo, para um elemento ou composto existente em um resíduo. Para Valocchi (1984) o fator de retardamento representa a defasagem entre a velocidade de avanço do soluto e a velocidade de avanço da frente de molhamento da solução percolante; desta forma, sendo o fator de retardamento um parâmetro que, indiretamente, expressa a capacidade do solo em reter íons, fica clara sua dependência em relação às interações entre a fase líquida e a fase sólida, durante a percolação da solução no solo.

Segundo van Genuchten et al. (1974) o coeficiente de dispersão-difusão, também denominado coeficiente de dispersão hidrodinâmica, coeficiente de difusão aparente ou dispersão longitudinal, é um parâmetro físico da equação do transporte de solutos. Ele expressa dois fenômenos de transporte aditivos: a dispersão mecânica, que representa o movimento diferenciado de solutos no solo, proporcionado por variações na velocidade de deslocamento da solução dentro dos poros individuais e entre poros de diferentes formas, diâmetros médios e direções, e a difusão iônica, que é o movimento térmico natural de constituintes dissolvidos e que ocorre em razão da existência de gradientes de concentração.

O estudo do efeito da competição catiônica sobre os valores do fator de retardamento e coeficiente de dispersão-difusão, parâmetros de entrada da equação de transporte dispersivoconvectivo, é fundamental para a correta adequação dos modelos de simulação do deslocamento dos solutos no solo. Em razão disto, este trabalho teve como objetivo avaliar os efeitos da competição catiônica nos valores do fator de retardamento $\left(f_{\mathrm{r}}\right)$ e do coeficiente de dispersão-difusão (D) dos metais zinco e cobre no solo.

\section{MATERIAL E MÉTODOS}

Tubos de PVC com 4,7 cm de diâmetro interno e $20 \mathrm{~cm}$ de comprimento, foram utilizados para acondicionamento de materiais de solo dos horizontes A, B e C de um Latossolo
Vermelho-Amarelo, álico (LVa) e um Podzólico VermelhoAmarelo, câmbico, $(\mathrm{PVc})$ coletados no município de Viçosa, MG. As características físicas e químicas dos solos utilizados, determinadas conforme EMBRAPA (1997) estão apresentadas nas Tabelas 1 e 2, respectivamente.

Os materiais de solo foram acondicionados dentro dos tubos, obtendo-se colunas de solo com $15 \mathrm{~cm}$ de altura. As colunas de solo, após compactação detalhada por Ramos (1998) foram saturadas por capilaridade durante $72 \mathrm{~h}$. Por meio da aplicação de água deionizada, sob carga hidrostática constante e igual a $4 \mathrm{~cm}$, obteve-se a condição de escoamento permanente, após a qual se cessou a aplicação de água e, terminada a infiltração completa da água, iniciou-se a aplicação da solução contaminante, sob idêntica carga hidrostática. Como contaminantes, foram utilizadas soluções mono-espécies (200 mg L-1 de Cu; $700 \mathrm{mg} \mathrm{L}^{-1}$ de $\mathrm{Zn}$ ) e multi-espécies (os dois elementos juntos nas mesmas concentrações). Os efluentes foram coletados, sistematicamente, até se atingir um volume equivalente a quinze volumes de poros. Os três primeiros volumes de poros de efluentes das colunas de lixiviação que receberam solução mono-espécie de Zn e multi-espécie, foram coletados em recipientes equivalentes a 0,25 volume de poros, devido à mobilidade deste metal, e nas colunas que receberam solução mono-espécie de $\mathrm{Cu}$ coletaram-se efluentes a cada volume de poros unitário.

Nas soluções mono-espécies considerou-se que os metais em estudo não ficaram expostos à competição proporcionada por outros cátions, razão pela qual as condições de interação com a matriz do solo foram denominadas de "sem competição". No caso da solução multi-espécie, um metal ficou submetido à competição do outro, sendo suas condições de interação com o meio denominadas "com competição".

Curvas de eluição foram plotadas com os dados obtidos, considerando-se concentração relativa $\left(\mathrm{C} / \mathrm{C}_{0}\right.$, em que $\mathrm{C}$ é a concentração do efluente coletado e $\mathrm{C}_{0}$ a concentração inicial da solução contaminante) versus volume de efluente coletado (em unidade de volume de poros). Com base nos dados obtidos, fez-se o cálculo do $\mathrm{f}_{\mathrm{r}}$ e $\mathrm{D}$ pelo método da Análise dos Mínimos

Tabela 1. Caracterização física das amostras de solo acondicionadas nas colunas utilizadas nos testes

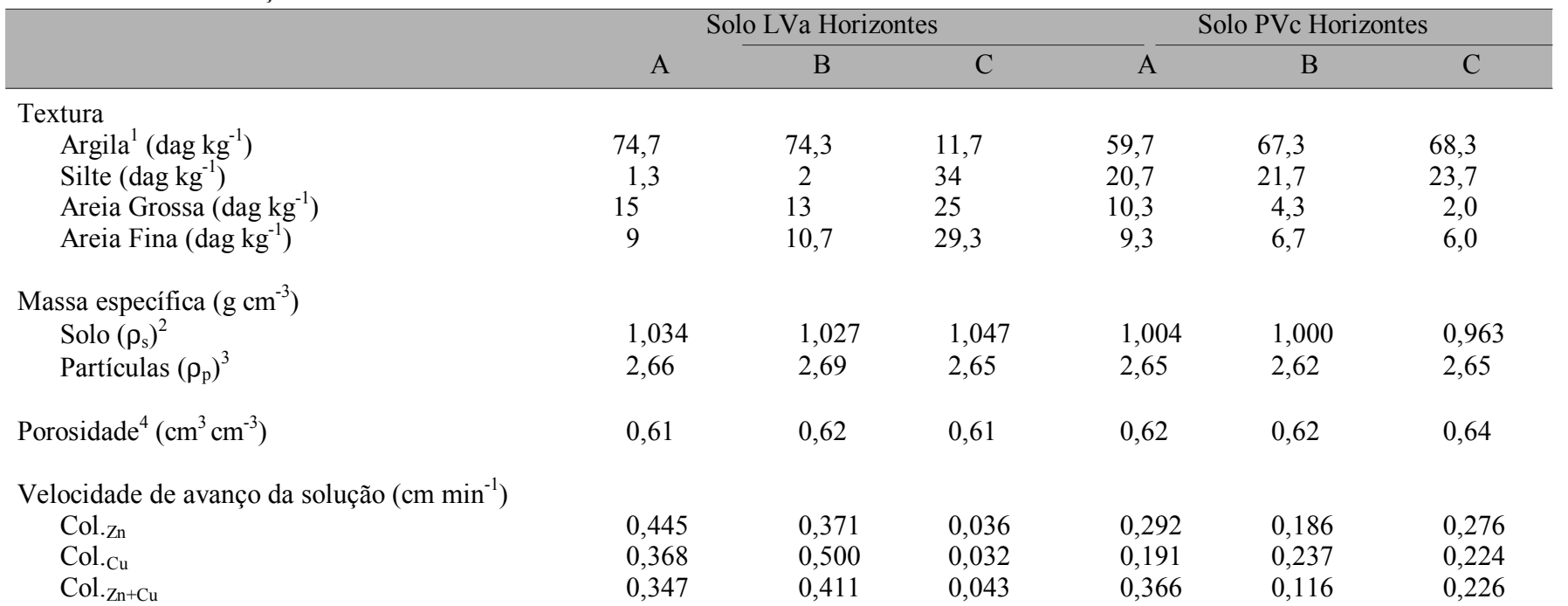

\footnotetext{
Método da Pipeta

Método da Proveta

Método do Balão Volumétrico

${ }^{4}$ Porosidade $\left(\varepsilon=1-\rho_{\mathrm{s}} / \rho_{\mathrm{p}}\right)$
} 
Tabela 2. Caracterização química das amostras de solos estudados

\begin{tabular}{|c|c|c|c|c|c|c|}
\hline \multirow{2}{*}{ Características } & \multicolumn{3}{|c|}{ Solo LVa Horizontes } & \multicolumn{3}{|c|}{ Solo PVc Horizontes } \\
\hline & A & $\mathrm{B}$ & $\mathrm{C}$ & A & B & $\mathrm{C}$ \\
\hline $\mathrm{pH}^{1}$ & 4,61 & 5,06 & 5,38 & 6,26 & 5,36 & 5,88 \\
\hline \multicolumn{7}{|c|}{ Cátions trocáveis $\left(\mathrm{cmol}_{\mathrm{c}} \mathrm{kg}^{-1}\right)$} \\
\hline $\mathrm{Ca}^{++2}$ & 0,25 & 0,08 & 0,00 & 3,98 & 0,45 & 0,02 \\
\hline $\mathrm{Mg}^{++}$ & 0,07 & 0,02 & 0,02 & 1,15 & 0,22 & 0,08 \\
\hline $\mathrm{K}^{+3}$ & 0,10 & 0,02 & 0,02 & 0,51 & 0,09 & 0,04 \\
\hline $\mathrm{Na}^{+3}$ & 0,10 & 0,02 & 0,03 & 0,49 & 0,09 & 0,03 \\
\hline Soma de Bases ${ }^{4}$ & 0,52 & 0,14 & 0,07 & 6,09 & 0,85 & 0,17 \\
\hline $\mathrm{CTCe}^{5}$ & 2,32 & 0,94 & 0,72 & 6,19 & 1,35 & 0,57 \\
\hline $\mathrm{H}+\mathrm{Al}^{6}$ & 9,99 & 6,55 & 2,39 & 3,95 & 4,61 & 3,33 \\
\hline $\mathrm{MO}^{7}$ & 5,71 & 3,02 & 0,27 & 5,24 & 2,42 & 1,14 \\
\hline
\end{tabular}

${ }^{1} \mathrm{pH}$ em água

${ }^{2}$ Extrações com KCl, 1 mol.L $\mathrm{L}^{-1}$

${ }^{3}$ Extrações com Mehlich 1

${ }^{4}$ Soma de bases trocáveis

Capacidade de troca cationica efetiva, determinada pelo método da soma de bases trocáveis

${ }^{6}$ Acidez Potencial, determinada pelo método titulométrico

Matéria orgânica, determinada pelo método de Walkey Black (EMBRAPA, 1997)

Quadrados, utilizando-se o aplicativo CXTFIT - 1.0, desenvolvido por Parker \& van Genuchten (1984). Foram obtidos dados referentes a três repetições e a análise quantitativa dos mesmos foi realizada considerando-se os valores de $\mathrm{f}_{\mathrm{r}}$ e $\mathrm{D}$, calculados para todas as repetições do experimento.

\section{RESULTADOS E DISCUSSÃO}

Nas Figuras de 1 e 2 estão apresentadas as curvas de eluição do zinco e cobre, sem competição e com competição, obtidas experimentalmente.

Pode-se observar que, em todos os horizontes dos solos estudados, o cobre apresentou maiores valores de fator de retardamento que o zinco, confirmando Matos et al. (1998) que também encontraram maior interação do cobre que a observada para o zinco nesses solos. Os valores de $\mathrm{f}_{\mathrm{r}}$ e de $\mathrm{D}$ do zinco e do cobre diminuíram, independentemente do material de solo estudado, quando os cátions estiveram em competição na solução contaminante, o que pode ser observado analisando-se as curvas de eluição apresentadas nas Figuras 1 e 2. A diminuição nos valores do $f_{r}$ caracterizou-se pelo deslocamento, para a esquerda, das curvas de eluição de cada cátion, quando em competição. Esta maior facilidade de lixiviação pode ser atribuída ao fato de que, quando há mais de um cátion presente na solução contaminante, haverá menor disponibilidade de sítios de adsorção para ambos, o que facilita a sua presença na solução do solo, de acordo com a equação para determinação de D, apresentada por van Genuchten et al. (1974) ou seja:

$$
D=\frac{v L_{c}}{4 \pi f_{r}^{2} b^{2}}
$$

em que $v$ é a velocidade média de avanço da solução percolante nos poros $\left(\mathrm{L} \mathrm{T}^{-1}\right) ; \mathrm{L}_{\mathrm{c}} \mathrm{o}$ comprimento da coluna $(\mathrm{L}) \mathrm{e}$ b a declividade da curva de eluição $\left(\mathrm{L} \mathrm{L}^{-1}\right)$; para a concentração relativa igual a 0,5 , esperava-se um aumento nos valores de $\mathrm{D}$ com a redução
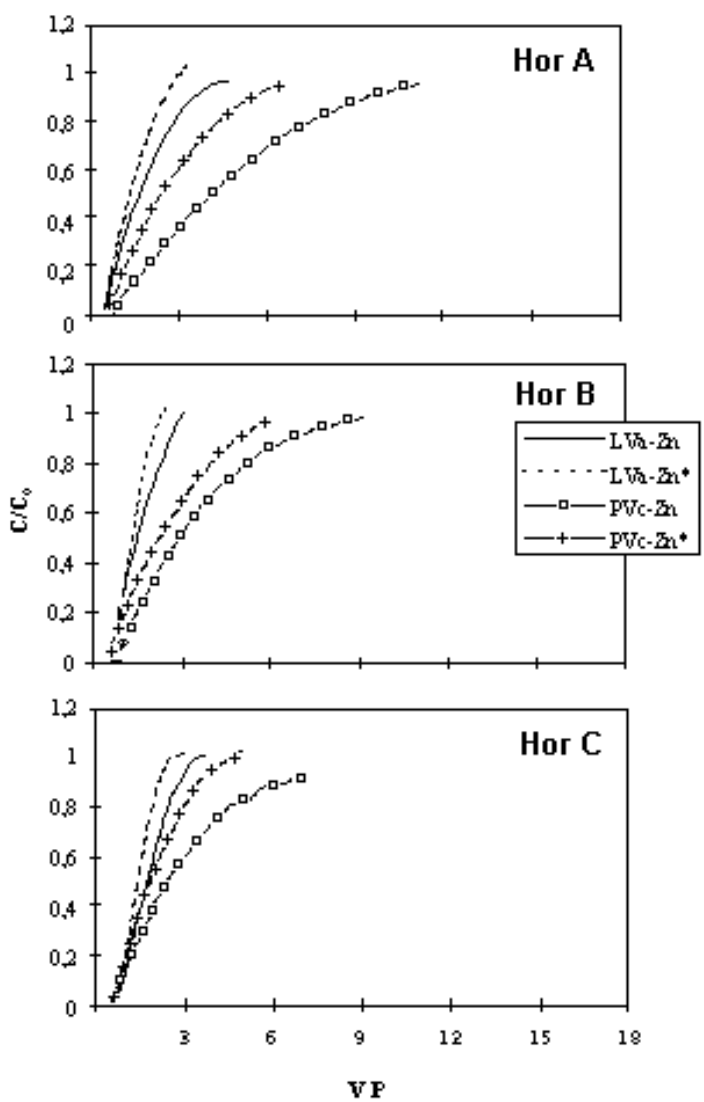

Figura 1. Curvas de eluição do zinco sem competição (Zn) e em competição ( $\mathrm{Zn} *$ ) nos horizontes A, B e C dos solos LVa e PVc

dos valores de $\mathrm{f}_{\mathrm{r}}$, o que, entretanto, não aconteceu, demonstrando independência entre esses dois parâmetros; percebeu-se, na verdade, maior influência do aumento da declividade das curvas de eluição, condicionada pela competição entre os cátions.

Os valores médios do fator de retardamento e do coeficiente de dispersão-difusão (Tabela 3) e os valores médios da velocidade de avanço da solução percolante (Tabela 1) ratificam 
Tabela 3. Valores médios do fator de retardamento (adimensional) e do coeficiente de dispersão-difusão do zinco e do cobre, com ou sem competição, calculados pelo método da análise dos mínimos quadrados

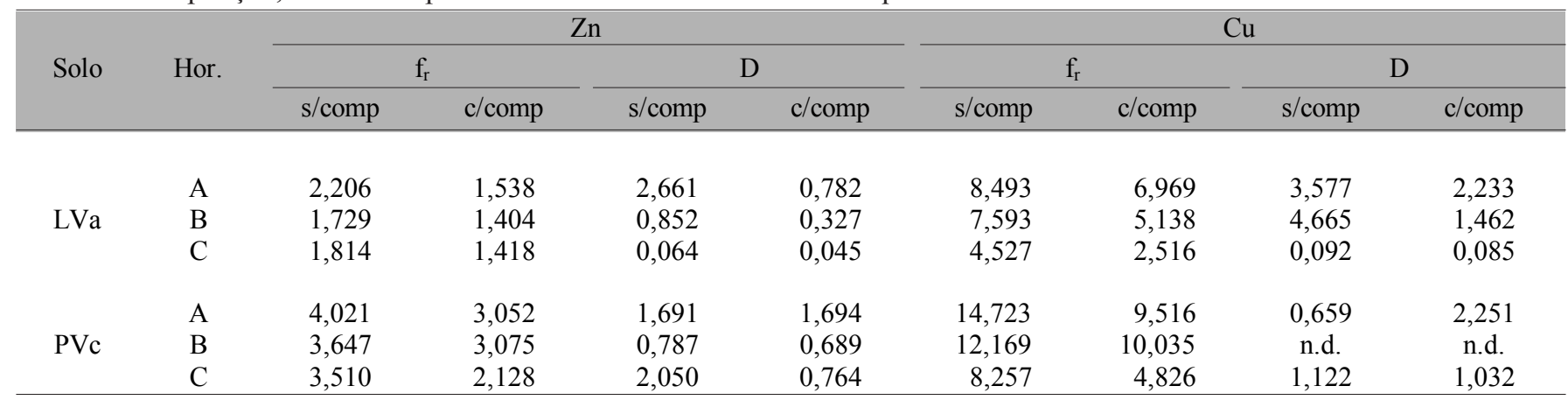
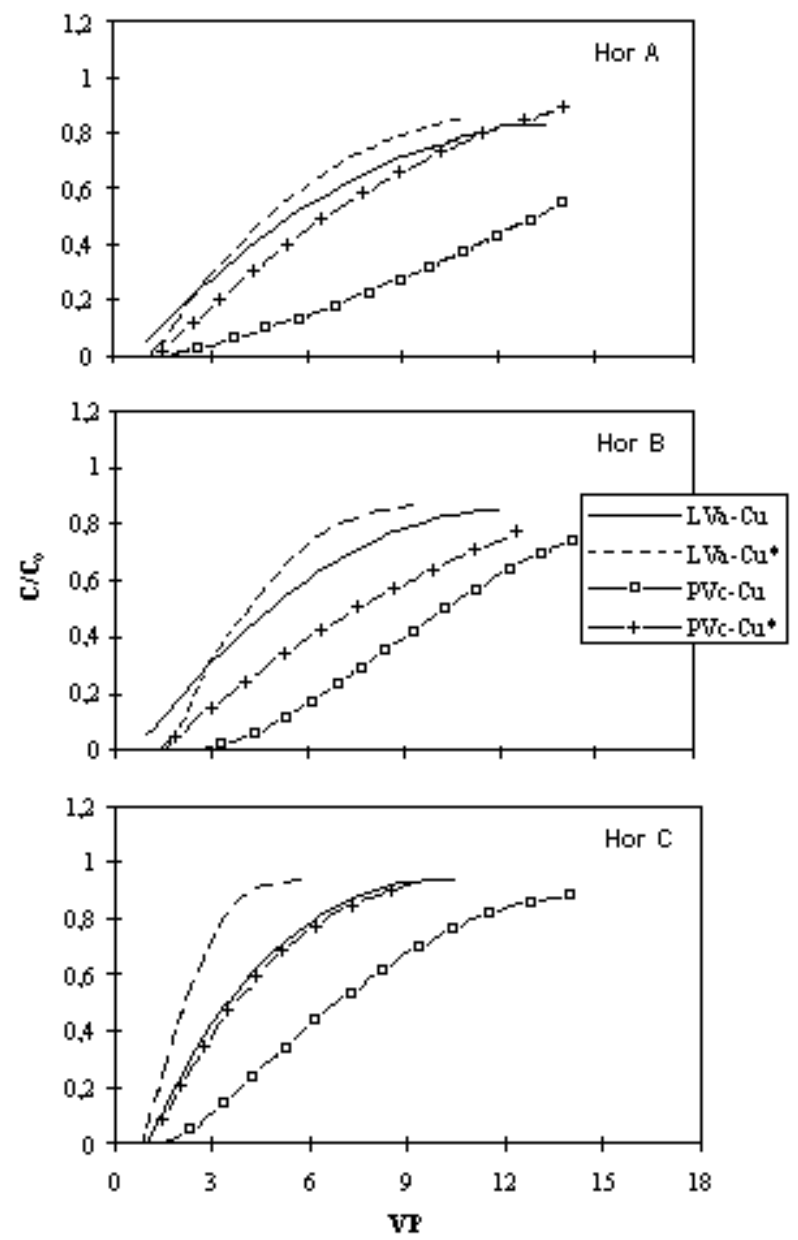

Figura 2. Curvas de eluição do cobre sem competição $(\mathrm{Cu})$ e em competição $\left(\mathrm{Cu}^{*}\right)$ nos horizontes A, B e C dos solos LVa e PVc

a análise qualitativa, realizada anteriormente, no que diz respeito à capacidade de retenção de cada material de solo. Percebe-se que, quando não há competição, o horizonte A do PVc apresenta maior capacidade de retenção dos dois cátions que os horizontes $\mathrm{B}$ e $\mathrm{C}$ do mesmo solo mas, quando os dois cátions estiveram presentes na solução, ou seja, quando houve competição entre eles, os valores de $\mathrm{f}_{\mathrm{r}}$ diminuíram mais que nos outros horizontes. Acredita-se que as mais baixas velocidades de avanço da solução contaminante, comumente encontradas no horizonte $\mathrm{C}$ dos solos estudados, tenham proporcionado, aos dois cátions, maior tempo de contato com os sítios de adsorção, o que conduziu à obtenção de valores relativamente altos para o fator de retardamento.

\section{CONCLUSÕES}

Os resultados obtidos permitiram concluir que:

1. Nos solos estudados, em todos os horizontes o cobre apresentou maiores valores de fator de retardamento que o zinco, indicando maior interação com a fração sólida do solo.

2. As baixas velocidades de avanço das soluções proporcionaram a obtenção de baixos valores de coeficiente de dispersão-difusão no solo.

3. A competição entre o zinco e cobre diminui a retenção individual de cada cátion nos materiais de solo estudados, o que tendeu a promover uma diminuição dos valores de fator de retardamento $\left(\mathrm{f}_{\mathrm{r}}\right)$ e do coeficiente de dispersão-difusão (D).

\section{LITERATURA CITADA}

Campos, T.M.; Elbachá, A.T. Avaliação do fator de retardamento por adsorção no transporte de zinco em solos argilosos. In: Simpósio sobre Barragens de Rejeitos e Disposição de Resíduos-Regeo'91, Rio de Janeiro, 1991. Anais... Rio de Janeiro, 1991. p.271-282.

Corrêa, M.M. Mobilidade e retenção do inseticida-nematicida sulfona de aldicarbe em colunas de solos de regiões produtoras de batata de Minas Gerais. Lavras, MG: UFLA, 1996. 76p. Dissertação Mestrado

EMBRAPA - Empresa Brasileira de Pesquisa Agropecuária. Manual de métodos de análise de solo. Rio de Janeiro: Centro Nacional de Pesquisas de Solos/CNPS, 1997. 212p. Documento 1

Ferreira, M.E.; Cruz, M.C.P. da. Micronutrientes no solo: Cobre. In: Ferreira, M.E.; Cruz, M.C.P. da. (ed) Micronutrientes na Agricultura. Piracicaba, SP: Associação Brasileira para Pesquisa da Potassa e do Fosfato/CNPq, 1991, p.131-151.

Matos, A.T. Fatores de retardamento e coeficientes de dispersão-difusão do zinco, cádmio, cobre e chumbo, em solos do município de Viçosa, MG. Viçosa, MG, UFV, 1995. 110p. Tese de Doutorado.

Matos, A.T.; Costa, L.M. da; Fontes, M.P.F.; Martinez, M.A. Correlação entre os fatores de retardamento e coeficientes de dispersão-difusão do zinco, cádmio, cobre e chumbo, e algumas propriedades físicas dos solos. Engenharia na Agricultura, Viçosa, v.6, n.4, p.235-246, 1998.

Parker, J.C.; van Genuchten, M. Th. Determining transport parameters from laboratory and field tracer experiments. Blacksburg: Virginia Agricultural Experiment Station. 1984. 96p. Bulletin 84-3. 
Ramos,V.B.N. Valores de fatores de retardamento e coeficientes de dispersão-difusão de zinco e cobre, obtidos usando-se soluções mono e multi-espécies. Viçosa: U.F.V., 1998. 66 p. Dissertação Mestrado

Valocchi, A.J. Describing the transport of ion-exchanging contaminants using an effective $\mathrm{Kd}$ approach. Water Resource Research, Washington, v.20, n.4, p.499-503, 1984. van Genuchten, M.Th.; van Davidson, J.M.; Wierenga, P.J. An evaluation of kinetic and equilibrium equations for the prediction of pesticide movement through porous media. Soil Science Society of America Proceedings, Madison, n.38, p.29-35, 1974. van Genuchten, M.Th.; Wierenga, P.J. Solute dispersion coefficients and retardation factors. In: Klute, A. (ed.) Methods of soil analysis. I: Physical and mineralogical methods. Madison: ASA, SSSA, 1986, p.1025-1054. 